\title{
On the Decomposition of Generalized Semiautomata
}

\author{
MERVE NUR CAKIR, MEHWISH SALEEMI, KARL-HEINZ ZIMMERMANN \\ Department of Computer Engineering \\ Hamburg University of Technology \\ 20171 Hamburg \\ GERMANY
}

\begin{abstract}
Semiautomata are abstractions of electronic devices that are deterministic finite-state machines having inputs but no outputs. Generalized semiautomata are obtained from stochastic semiautomata by dropping the restrictions imposed by probability. It is well-known that each stochastic semiautomaton can be decomposed into a sequential product of a dependent source and deterministic semiautomaton making partly use of the celebrated theorem of Birkhoff-von Neumann. It will be shown that each generalized semiautomaton can be partitioned into a sequential product of a generalized dependent source and a deterministic semiautomaton.
\end{abstract}

Key-Words: - Semiautomaton, stochastic automaton, monoid, Birkhoff-von Neumann

Received: October 1, 2020. Revised: May 4, 2021. Accepted: May 22, 2021. Published: June 9, 2021.

\section{Introduction}

The theory of discrete stochastic systems has been initiated by the work of Shannon [14] and von Neumann [10]. While Shannon has considered memoryless communication channels and their generalization by introducing states, von Neumann has studied the synthesis of reliable systems from unreliable components. The fundamental work of Rabin and Scott [12] about deterministic finite-state automata has led to two generalizations. First, the generalization of transition functions to conditional distributions studied by Carlyle [3] and Starke [15]. This in turn yields a generalization of discrete-time Markov chains in which the chains are governed by more than one transition probability matrix. Second, the generalization of regular sets by introducing stochastic automata as described by Rabin [11].

By the work of Turakainen [16], stochastic acceptors can be viewed equivalently as generalized automata in which the "probability" is neglected. This leads to a more accessible approach to stochastic automata [5].

On the other hand, the class of nondeterministic automata [13] can be generalized to monoidal automata, where the input alphabet corresponds to an arbitrary monoid instead of a free monoid [8, 9, 17]. This leads to the class of monoidal automata whose languages are closed under a smaller set of operations when compared with regular languages.

A first step into the study of automata theory are semiautomata which are abstractions of electronic devices that are deterministic finite-state machines having inputs but no outputs [7, 9]. Generalized semiautomata are obtained from stochastic semiautomata by dropping the restrictions imposed by probabil- ity [5, 16]. It is well-known that each stochastic automaton can be decomposed into a sequential product of a dependent source and deterministic semiautomaton [2]. This result makes use in part of the celebrated theorem of Birkhoff-von Neumann that each doubly stochastic matrix can be represented as a convex combination of permutation matrices. In this paper, it will be shown that each generalized semiautomaton can be partitioned into a sequential product of a generalized dependent source and a deterministic semiautomaton.

Notation. Let $X$ be a set. The set of all mappings on $X, T(X)=\{f \mid f: X \rightarrow X\}$, forms a monoid under function composition $(f g)(x)=g(f(x)), x \in$ $X$, and the identity function $\operatorname{id}_{X}: X \rightarrow X: x \mapsto x$ is the identity element. The monoid $T(X)$ is called the full transformation monoid of $X$.

\section{Semiautomata}

Semiautomata are abstractions of electronic devices which are deterministic finite-state machines having input but no output [7, 9].

A (deterministic) semiautomaton (SA) is a triple

$$
A=\left(S, \Sigma,\left\{\delta_{x} \mid x \in \Sigma\right\}\right)
$$

where

- $S$ is the non-empty finite set of states,

- $\Sigma$ is the set of input symbols,

- $\delta_{x}: S \rightarrow S$ is a (partial) mapping for each $x \in \Sigma$.

Let $\Sigma^{*}$ denote the free monoid over the alphabet $\Sigma$. By the universal property of free monoids [4, 9], the mapping $\delta: \Sigma \rightarrow T(S): x \mapsto \delta_{x}$ extends uniquely to 
a monoid homomorphism $\delta: \Sigma^{*} \rightarrow T(S): u \mapsto \delta_{u}$ such that for each word $u=x_{1} \ldots x_{k} \in \Sigma^{*}$,

$$
\delta_{u}=\delta_{x_{1}} \cdots \delta_{x_{k}}
$$

and particularly $\delta_{\epsilon}=\mathrm{id}_{S}$. The mapping $\delta$ is called the transition function of $A$. Its image $T(A)=\left\{\delta_{u} \mid\right.$ $\left.u \in \Sigma^{*}\right\}$ is a submonoid of the full transformation monoid $T(S)$ generated by $\left\{\delta_{x} \mid x \in \Sigma\right\}$. The semiautomaton $A$ is also denoted by $A=(S, M, \delta)$ or $A=\left(S^{A}, M^{A}, \delta^{A}\right)$.

A semiautomaton $A=(S, \Sigma, \delta)$ serves as a skeleton of a deterministic finite-state machine that is exactly in one state at a time. If the semiautomaton $A$ is in state $s$ and reads the word $u \in \Sigma^{*}$, it transits into the state $s^{\prime}=\delta_{u}(s)$.

Example 1. Consider the semiautomaton $A=$ $(S, \Sigma, \delta)$ with state set $S=\{1,2,3\}$, input alphabet $\Sigma=\{x, y\}$, and transition function $\delta$ given by the automaton graph in Fig. 1. The associated transformation monoid is generated by the transformations

$$
\delta_{x}=\left(\begin{array}{ccc}
1 & 2 & 3 \\
1 & 1 & 1
\end{array}\right) \quad \text { and } \quad \delta_{y}=\left(\begin{array}{lll}
1 & 2 & 3 \\
2 & 2 & 3
\end{array}\right) .
$$

We have

$$
\begin{aligned}
\delta_{x x}=\left(\begin{array}{ccc}
1 & 2 & 3 \\
1 & 1 & 1 \\
1 & 2 & 3 \\
1 & 1 & 1
\end{array}\right), & \delta_{x y}=\left(\begin{array}{lll}
1 & 2 & 3 \\
2 & 2 & 2 \\
1 & 2 & 3 \\
2 & 2 & 3
\end{array}\right),
\end{aligned}
$$

Hence, the transformation monoid $T(A)$ is given by $\left\{\operatorname{id}_{S}, \delta_{x}, \delta_{y}, \delta_{x y}\right\}$.

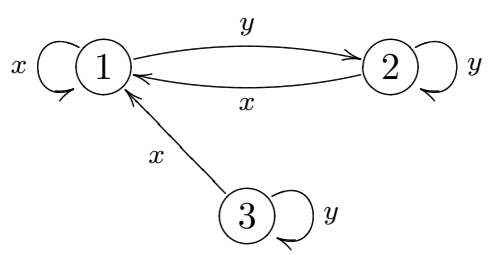

Figure 1: Semiautomaton.

\section{Generalized Semiautomata}

Stochastic automata are a generalization of nondeterministic finite state automata [5]. Generalized automata can be obtained from stochastic automata by dropping the restrictions imposed by probability $[5,16,17]$.

A generalized semiautomaton (GSA) is a triple

$$
A=\left(S, \Sigma,\left\{Q_{x} \mid x \in \Sigma\right\}\right),
$$

where
- $S$ is the non-empty finite set of states,

- $\Sigma$ is the input alphabet, and

- $Q$ is a collection of $n \times n$ nonnegative matrices $Q_{x}, x \in \Sigma$, where $n$ is the number of states.

In view of the universal property of free monoids [4, 9], the mapping $Q: \Sigma \rightarrow \mathbb{R}^{n \times n}$ : $x \mapsto Q_{x}$ extends uniquely to a monoid homomorphism $Q: \Sigma^{*} \rightarrow \mathbb{R}^{n \times n}$ such that for each word $u=x_{1} \ldots x_{k} \in \Sigma^{*}$,

$$
Q_{u}=Q_{x_{1}} \cdots Q_{x_{k}}
$$

and particularly $Q_{\epsilon}=I_{n}$ is the $n \times n$ identity matrix. The mapping $Q$ is called the transition function of $A$. Its image $T(A)=\left\{Q_{u} \mid u \in \Sigma^{*}\right\}$ is a submonoid of the full transformation monoid $T(S)$ generated by $\left\{Q_{x} \mid x \in \Sigma\right\}$. The generalized semiautomaton $A$ is also denoted by $A=(S, \Sigma, Q)$ or $A=\left(S^{A}, \Sigma^{A}, Q^{A}\right)$.

The state set $S=\left\{s_{1}, \ldots, s_{n}\right\}$ can be viewed as the standard basis for the Euclidean vector space $\mathbb{R}^{n}$, where $s_{i}$ is the basis vector whose $i$ th coordinate is 1 and all others are 0 . In this way, the $(i, j)$ the entry of the matrix $Q_{u}=\left(s_{i j}^{(u)}\right)$ is given by $s_{i j}^{(u)}=s_{i}^{T} Q_{u} s_{j}$.

Proposition 1. Each deterministic semiautomaton is a generalized automaton.

Proof. Let $A=(S, \Sigma, \delta)$ be a deterministic semiautomaton and let $S=\left\{s_{1}, \ldots, s_{n}\right\}$. Define the generalized semiautomaton $B=(S, \Sigma, Q)$, where for each $x \in \Sigma$, the $(i, j)$ th entry of $Q_{x}$ is 1 if $\delta_{x}\left(s_{i}\right)=s_{j}$ and otherwise 0 . Then the mapping $T(A) \rightarrow T(B)$ : $\delta_{u} \mapsto Q_{u}$ is a monoid isomorphism.

A generalized semiautomaton $A=(S, \Sigma, P)$ is called stochastic if the matrices $P_{x}, x \in \Sigma$, are stochastic, i.e., $P_{x}$ is a matrix of nonnegative real numbers such that each row sum is equal to 1 . The product of stochastic matrices is again a stochastic matrix and so the transition monoid $T(A)$ consists of the stochastic matrices $P_{u}, u \in \Sigma^{*}$. In particular, the $(i, j)$ th element $p\left(s_{j} \mid u, s_{i}\right)$ of the matrix $P_{u}$ is the transition probability that the automaton enters state $s_{j}$ when started in state $s_{i}$ and reading the word $u$.

Example 2. Let $m \geq 2$ be an integer. Put $\Sigma=$ $\{0, \ldots, m-1\}$. The stochastic semiautomaton $\mathcal{A}=$ $\left(\left\{s_{1}, s_{2}\right\}, \Sigma, P\right)$ given by

$$
P_{x}=\frac{1}{m}\left(\begin{array}{cc}
m-x & x \\
m-x-1 & x+1
\end{array}\right), \quad x \in \Sigma,
$$

is called $m$-adic semiautomaton. For each word $u=$ $x_{1} \ldots x_{k} \in \Sigma^{*}$,

$$
P_{u}=\frac{1}{m^{k}}\left(\begin{array}{cc}
m^{k}-w_{k} & w_{k} \\
m^{k}-w_{k}-1 & w_{k}+1
\end{array}\right),
$$


where $w_{k}=x_{k} m^{k-1}+\ldots+x_{2} m+x_{1}$ and the entry $\frac{1}{m^{k}} w_{k}$ corresponds in the m-adic representation to $0 . x_{k} \ldots x_{1}$.

A generalized semiautomaton $A=(S, \Sigma, D)$ is called doubly stochastic if the matrices $D_{x}, x \in \Sigma$, are doubly stochastic, i.e., $D_{x}$ is a matrix of nonnegative real numbers such that each row and column sum is equal to 1 . The product of doubly stochastic matrices is again a doubly stochastic matrix and so the transition monoid $T(A)$ consists of the doubly stochastic matrices $D_{u}, u \in \Sigma^{*}$.

\section{Decomposition of Generalized Semiautomata}

The objective is to decompose each generalized semiautomata into a sequential product of a generalized dependent source and a deterministic semiautomaton. The corresponding result for stochastic semiautomata has been proved by Bukharaev [2].

A generalized dependent source is a triple

$$
\Gamma=(\Sigma, \Xi,\{\gamma(z \mid x) \mid x \in \Sigma, z \in \Xi\}),
$$

where $\Sigma$ and $\Xi$ are alphabets and $\gamma: \Sigma \times \Xi \rightarrow \mathbb{R}_{\geq 0}$ : $(x, z) \rightarrow \gamma(z \mid x)$ is a mapping which is extended recursively to $\Sigma^{*} \times \Xi^{*}$ as follows:

$$
\begin{aligned}
& \text { - } \gamma(\epsilon \mid \epsilon)=1, \\
& \text { - } \gamma(v \mid u)=0 \text { for all } u \in \Sigma^{*} \text { and } v \in \Xi^{*} \text { with } \\
& |u| \neq|v| \text {, and } \\
& \text { - } \gamma(z v \mid x u)=\gamma(x \mid z) \gamma(u \mid v) \text { for all } x \in \Sigma, \\
& \\
& u \in \Sigma^{*}, z \in \Xi \text { and } v \in \Xi^{*} \text {. }
\end{aligned}
$$

A generalized dependent source $\Gamma$ is also denoted by $\Gamma=(\Sigma, \Xi, \gamma)$

In particular, a dependent source is a generalized dependent source $\Gamma=(\Sigma, \Xi, \gamma)$, where $\Sigma$ and $\Xi$ are alphabets and for each $x \in \Sigma, \gamma(\cdot \mid x)$ defines a (conditional) probability measure on $\Xi$. This measure can be extended for each $u \in \Sigma^{*}$ to a (conditional) probability measure $\gamma(\cdot \mid u)$ on $\Xi^{*}$ along the same lines as above. Note that a dependent source can be viewed as a stochastic input-output automaton with a single state [2, 5].

The sequential product of generalized dependent source $\Gamma=(\Sigma, \Xi, \gamma)$ and generalized semiautomaton $B=\left(S, \Xi, Q^{B}\right)$ defines a generalized semiautomaton $A=\left(S, \Sigma, Q^{A}\right)$ such that for all $x \in \Sigma$,

$$
Q_{x}^{A}=\sum_{z \in \Xi} \gamma(z \mid x) \cdot Q_{z}^{B}
$$

By induction, for all $u \in \Sigma^{*}$,

$$
Q_{u}^{A}=\sum_{v \in \Xi^{*}} \gamma(v \mid u) \cdot Q_{v}^{B} .
$$

A permutation matrix $P$ is a square binary matrix which has exactly one entry of 1 in each row and each column and 0's elsewhere. By the Birkhoff-von Neumann theorem [6], for each $n \times n$ doubly stochastic matrix $P$ there exist real numbers $\alpha_{1}, \ldots, \alpha_{N} \geq$ 0 with $\sum_{i=1}^{N} \alpha_{i}=1$ and permutation matrices $P_{1}, \ldots, P_{N}$ such that

$$
P=\alpha_{1} P_{1}+\ldots+\alpha_{N} P_{N}
$$

This representation is also known as Birkhoff-von Neumann decomposition. Such a representation of a doubly stochastic matrix as a convex combination of permutation matrices may not be unique. By the Marcus-Ree Theorem [1], $N \leq n^{2}-2 n+2$ for dense matrices.

A square matrix $P$ is called deterministic if it has exactly one entry of 1 in each row and 0's elsewhere. In particular, each permutation matrix is deterministic. For each $n \times n$ stochastic matrix $P$ there exist real numbers $\alpha_{1}, \ldots, \alpha_{N} \geq 0$ with $\sum_{i=1}^{N} \alpha_{i}=1$ and deterministic matrices $P_{1}, \ldots, P_{N}$ such that

$$
P=\alpha_{1} P_{1}+\ldots+\alpha_{N} P_{N}
$$

Such a representation of a stochastic matrix as a convex combination of deterministic matrices may not be unique.

A square matrix $P$ is called semideterministic if in each nonzero row there is exactly one entry of 1 and 0 's elsewhere. In particular, each deterministic matrix is semideterministic.

Proposition 2. For each nonnegative square matrix $A$, there exist real numbers $\alpha_{1}, \ldots, \alpha_{N} \geq 0$ and semideterministic matrices $P_{1}, \ldots, P_{N}$ such that

$$
A=\alpha_{1} P_{1}+\ldots+\alpha_{N} P_{N}
$$

Proof. For each nonnegative square matrix $P=\left(p_{i j}\right)$ let $p_{i, \pi(i)}$ be a minimal nonzero entry in row $i$. Consider the semideterministic matrix $D=\left(d_{i j}\right)$ with $d_{i, \pi(i)}=1$ for each $i$ and $d_{i j}=0$ otherwise. Moreover, put $m(P)=\min \left\{p_{i j} \mid p_{i j} \neq 0\right\}$. Then $P-m(P) D$ is a nonnegative matrix with at least one more zero entry than $P$. Iterating this step a finite number $N$ of times gives a sequence $\left(P_{k}\right)_{1 \leq k \leq N}$ of nonnegative matrices and a sequence $\left(D_{k}\right)_{1 \leq k \leq N}$ of semideterministic matrices such that $P_{1}=A$, $P_{k+1}=P_{k}-m\left(P_{k}\right) D_{k}$ for $1 \leq k \leq N$, and $P_{N+1}=0$. This yields the decomposition of $A$ as a linear combination of semideterministic matrices $A=\sum_{k=1}^{N} m\left(P_{k}\right) D_{k}$.

For doubly stochastic and stochastic matrices, the proof is similar. 
Example 3. Consider the nonnegative matrix

$$
A=\left(\begin{array}{lll}
2 & 4 & 6 \\
2 & 2 & 8 \\
3 & 3 & 6
\end{array}\right)
$$

A sequence of reductions showing the selected entries at each step is

$$
\begin{gathered}
\left(\begin{array}{ccc}
\underline{2} & 4 & 6 \\
\underline{2} & 2 & 8 \\
\underline{3} & 3 & 6
\end{array}\right),\left(\begin{array}{ccc}
0 & \underline{4} & 6 \\
0 & \underline{2} & 8 \\
\underline{1} & \frac{3}{3} & 6
\end{array}\right), \\
\left(\begin{array}{ccc}
0 & \underline{3} & 6 \\
0 & \underline{1} & 8 \\
0 & \underline{3} & 6
\end{array}\right),\left(\begin{array}{ccc}
0 & \underline{2} & 6 \\
0 & 0 & \underline{8} \\
0 & \underline{2} & 6
\end{array}\right),\left(\begin{array}{ccc}
0 & 0 & \underline{6} \\
0 & 0 & \underline{6} \\
0 & 0 & \underline{6}
\end{array}\right),
\end{gathered}
$$

yields the decomposition

$$
\begin{aligned}
A= & 2\left(\begin{array}{lll}
1 & 0 & 0 \\
1 & 0 & 0 \\
1 & 0 & 0
\end{array}\right)+1\left(\begin{array}{lll}
0 & 1 & 0 \\
0 & 1 & 0 \\
1 & 0 & 0
\end{array}\right) \\
& +1\left(\begin{array}{lll}
0 & 1 & 0 \\
0 & 1 & 0 \\
0 & 1 & 0
\end{array}\right)+2\left(\begin{array}{lll}
0 & 1 & 0 \\
0 & 0 & 1 \\
0 & 1 & 0
\end{array}\right) \\
& +6\left(\begin{array}{lll}
0 & 0 & 1 \\
0 & 0 & 1 \\
0 & 0 & 1
\end{array}\right)
\end{aligned}
$$

Theorem 3. Each generalized semiautomaton $A=$ $(S, \Sigma, Q)$ can be represented as a sequential product of a generalized dependent source $\Gamma=(\Sigma, \Xi, \gamma)$ and a semideterministic semiautomaton $B=(S, \Xi, \delta)$.

In particular, each stochastic (or strongly stochastic) semiautomaton $A=(S, \Sigma, P)$ can be represented as a sequential product of a dependent source $\Gamma=(\Sigma, \Xi, \gamma)$ and a deterministic (or permutation) semiautomaton $B=(S, \Xi, \delta)$.

Proof. Let $\left\{D_{1}, \ldots, D_{N}\right\}$ denote the collection of $n \times n$ semideterministic matrices. Put $\Xi=$ $\{1, \ldots, N\}$ and for each $x \in \Sigma$, write $Q_{x}$ as a conical combination of semideterministic matrices

$$
Q_{x}=\sum_{z \in \Xi} \alpha(z, x) D_{z}
$$

This defines the generalized dependent source $\Gamma=$ $(\Sigma, \Xi, \gamma)$, where for each $x \in \Sigma$ and $z \in \Xi$,

$$
\gamma(z \mid x)=\alpha(z, x)
$$

and the deterministic automaton $B=(S, \Xi, \delta)$, where for each $z \in \Xi$, the transition $\delta_{z}: S \rightarrow S$ is given by the matrix $D_{z}$ as in the proof of Prop. 11. Then we obtain for each $x \in \Sigma$,

$$
Q_{x}^{A}=\sum_{z \in \Xi} \gamma(z \mid x) Q_{z}^{B}
$$

The second part is clear from the above remarks.
Example 4. Consider the generalized semiautomaton

$$
A=\left(\left\{s_{1}, s_{2}\right\},\left\{x_{1}, x_{2}\right\},\left\{Q_{x_{1}}, Q_{x_{2}}\right\}\right),
$$

where

$$
Q_{x_{1}}=\left(\begin{array}{ll}
2 & 3 \\
1 & 0
\end{array}\right) \quad \text { and } \quad Q_{x_{2}}=\left(\begin{array}{ll}
1 & 2 \\
0 & 3
\end{array}\right)
$$

Then

$$
Q_{x_{1}}=\left(\begin{array}{ll}
1 & 0 \\
1 & 0
\end{array}\right)+\left(\begin{array}{ll}
1 & 0 \\
0 & 0
\end{array}\right)+3\left(\begin{array}{ll}
0 & 1 \\
0 & 0
\end{array}\right)
$$

and

$$
Q_{x_{2}}=\left(\begin{array}{ll}
1 & 0 \\
0 & 1
\end{array}\right)+2\left(\begin{array}{ll}
0 & 1 \\
0 & 1
\end{array}\right) \text {. }
$$

Put $\Xi=\left\{z_{1}, \ldots, z_{5}\right\}$ and

$$
\begin{aligned}
& D_{z_{1}}=\left(\begin{array}{ll}
1 & 0 \\
1 & 0
\end{array}\right), \quad D_{z_{2}}=\left(\begin{array}{ll}
1 & 0 \\
0 & 0 \\
0 & 1 \\
0 & 0 \\
D_{z_{3}} & =
\end{array}\right), \quad D_{z_{4}}=\left(\begin{array}{ll}
1 & 0 \\
0 & 1
\end{array}\right), \\
& D_{z_{5}}=\left(\begin{array}{ll}
0 & 1 \\
0 & 1
\end{array}\right) .
\end{aligned}
$$

Then

$Q_{x_{1}}=D_{z_{1}}+D_{z_{2}}+3 D_{z_{3}} \quad$ and $\quad Q_{x_{2}}=D_{z_{4}}+2 D_{z_{5}}$.

This gives the state transition table of the deterministic semiautomaton $B=(S, \Xi, \delta)$, where

$$
\begin{array}{c|ccccc}
\delta^{B} & z_{1} & z_{2} & z_{3} & z_{4} & z_{5} \\
\hline s_{1} & s_{1} & s_{1} & s_{2} & s_{1} & s_{2} \\
s_{2} & s_{1} & - & - & s_{2} & s_{2}
\end{array}
$$

and the transitions of the generalized dependent source $\Gamma=(\Sigma, \Xi, \gamma)$, where

\begin{tabular}{c|ccccc}
$\gamma$ & $z_{1}$ & $z_{2}$ & $z_{3}$ & $z_{4}$ & $z_{5}$ \\
\hline$x_{1}$ & 1 & 1 & 3 & 0 & 0 \\
$x_{1}$ & 0 & 0 & 0 & 1 & 2
\end{tabular}

Example 5. Reconsider the $m$-adic semiautomaton $\mathcal{A}=\left(\left\{s_{1}, s_{2}\right\}, \Sigma, P\right)$. For each $x \in \Sigma$,

$$
\begin{aligned}
P_{x}= & \frac{m-x-1}{m}\left(\begin{array}{ll}
1 & 0 \\
1 & 0
\end{array}\right)+\frac{1}{m}\left(\begin{array}{ll}
1 & 0 \\
0 & 1
\end{array}\right) \\
& +\frac{x}{m}\left(\begin{array}{ll}
0 & 1 \\
0 & 1
\end{array}\right) .
\end{aligned}
$$

Put $\Xi=\left\{z_{1}, z_{2}, z_{3}\right\}$ and

$$
\begin{aligned}
& D_{z_{1}}=\left(\begin{array}{ll}
1 & 0 \\
1 & 0
\end{array}\right), \quad D_{z_{2}}=\left(\begin{array}{ll}
1 & 0 \\
0 & 1
\end{array}\right), \\
& D_{z_{3}}=\left(\begin{array}{ll}
0 & 1 \\
0 & 1
\end{array}\right) .
\end{aligned}
$$


Then for each $x \in \Sigma$,

$$
P_{x}=\frac{m-x-1}{m} D_{z_{1}}+\frac{1}{m} D_{z_{2}}+\frac{x}{m} D_{z_{3}} .
$$

This provides the state transition table of the deterministic semiautomaton $B=(S, \Xi, \delta)$, where

\begin{tabular}{c|ccc}
$\delta^{B}$ & $z_{1}$ & $z_{2}$ & $z_{3}$ \\
\hline$s_{1}$ & $s_{1}$ & $s_{1}$ & $s_{2}$ \\
$s_{2}$ & $s_{1}$ & $s_{2}$ & $s_{2}$
\end{tabular}

and the transitions of the dependent source $\Gamma=$ $(\Sigma, \Xi, \gamma)$, where for each $x \in \Sigma$,

\begin{tabular}{c|ccc}
$\gamma$ & $z_{1}$ & $z_{2}$ & $z_{3}$ \\
\hline$x$ & $\frac{m-x-1}{m}$ & $\frac{1}{m}$ & $\frac{x}{m}$
\end{tabular}

\section{References:}

[1] M. Marcus, R. Ree, Diagonals of doubly stochastic matrices, Quarterly J. Math., Vol.10, No.1, 1959, pp. 296-302.

[2] R. G. Bukharaev, Theorie der stochastischen Automaten, Teubner, Stuttgart, 1995.

[3] J. W. Carlyle, Reduced forms for stochastic sequential machines, Journal Mathematical Analysis and Applications, Vol.7, No.2, 1963, pp. 167175.

[4] A. H. Clifford, G. B. Preston, The Algebraic Theory of Semigroups, Amer. Math. Soc., New York, 1961.

[5] V. Claus, Stochastische Automaten, Teubner, Stuttgart, 1971.

[6] A. S. Davis, Markov chains as random input automata, Amer. Math. Monthly, Vol.68, No.3, 1961, pp. 264-267.
[7] A. Ginzburg, Algebraic Theory of Automata, Academic Press, New York, 1968.

[8] V. Diekert, M. Kufleitner, B. Steinberg, The Krohn-Rhodes theorem and local divisors, Fundam. Inform., Vol.116, No.1-4, 2012, pp. 65-77.

[9] S. Mihov, K.U. Schulz, Finite-State Techniques, Cambridge Univ. Press, New York, 2019.

[10] J. von Neumann, Probabilistic Logics and Synthesis of Reliable Organisms from Unreliable Components, in: Automata Studies, C. Shannon and J. McCarthy (eds), Annals of Mathematical Studies, Vol.X, No.34, Princeton Univ. Press, Princeton, 1956, pp. 43-98.

[11] M. O. Rabin, Probabilistic automata, Information and Control, Vol.6, No.3, 1963, pp. 230-245.

[12] M. O. Rabin, D. Scott, Finite automata and their decision problems, IBM Journal Research Development, Vol.3, No.3, 1959, pp. 114-125.

[13] A. Salomaa, Theory of Automata, Pergamon Press, Oxford, 1969.

[14] C. E. Shannon, The mathematical theory of communication, Bell System Technical Journal, Vol.5, No.1, 1948, pp. 379-423.

[15] P. H. Starke, Stochastische Ereignisse und Wortmengen, Zeitschrift für Mathematische Logik und Grundlagen der Mathematik, Vol.12, No.X, 1966, pp. 61-68.

[16] P. Turakainen, Generalized automata and stochastic languages, Proc. Amer. Math. Soc., Vol.21, No.2, 1969, pp. 303-309.

[17] M. N. Cakir, K.-H. Zimmermann, On stochastic automata over monoids, TU Hamburg, arxiv:2002.01214, 2020. 